\title{
A sensibilidade na medicina - um trajeto do exame à escuta,
} pela mão da medicina narrativa

Sensitivity in medicine - a path from examination to listening,

through the hand of narrative medicine.

La sensibilidad en la medicina: un camino desde el examen hasta la escucha, a través de la mano de la medicina narrativa.

\author{
Munira Aiex Proença ${ }^{1}$ \\ Ana Ribeiro Rosa ${ }^{2}$ \\ Agnes Cruvinel $^{3}$ \\ Manuela Rodrigues Müller ${ }^{4}$
}

\begin{abstract}
Resumo: A formação médica esteve centrada nas disciplinas técnico-científicas, privilegiando o raciocínio científico e as intervenções clínico-cirúrgicas. Ainda que as inovações curriculares denotem a necessidade de reformulação deste modelo, reorientando-o para uma formação mais generalista e humanista, perpetua-se um ensino pautado pelas tecnologias duras. $\mathrm{O}$ desenvolvimento das aptidões propedêuticas pouco valoriza o despertar da sensibilidade para aspectos subjetivos e relacionais. Este trabalho pretende evidenciar a importância da sensibilidade na formação médica pela inclusão da competência narrativa nos currículos médicos. Argumentamos que o cuidado integral, ampliado, se constrói pela integração da sensibilidade para os elementos biológicos, subjetivos e relacionais do processo saúde-doençabusca de cuidado, num enredo que dá sentido à experiência do adoecimento. Quanto melhor os clínicos souberem ouvir, absorver e interpretar os relatos que lhes trazem, melhor poderão estabelecer uma conexão que promova a mudança, cumprindo o cuidado.
\end{abstract}

Palavras-chave: encontro clínico, educação médica, empatia, humanidades, medicina narrativa.

\begin{abstract}
Medical education has been centered on technical-scientific disciplines, privileging scientific reasoning and clinical-surgical interventions. Although curricular innovations denote the need to reformulate this model into a more generalist and humanist education, hard technologies framework are still relevant in teaching. In developing propaedeutic skills, sensitivity to subjective and relational aspects is undervalued. This work intends to highlight the importance of sensitivity in medical education through the inclusion of narrative competence in medical curricula. We argue that comprehensive, expanded care is set out by merging sensitivity with the biological, subjective and relational elements of the health-disease-seeking care process, in a plot that gives meaning to the illness experience. The better clinicians know how to listen, absorb and interpret the reports brought to them, the better they may establish a connection that promotes change, improving care.
\end{abstract}

Keywords: empathy, humanities, medical schools, narrative medicine, physician-patient relations.

Resumen: La educación médica se centró en disciplinas técnico-científicas, privilegiando el razonamiento científico y las intervenciones clínico-quirúrgicas. Si bien las innovaciones curriculares denotan la necesidad de reformular este modelo, reorientándolo hacia una educación más generalista y humanista, se perpetúa una enseñanza guiada por tecnologías duras. El desarrollo de habilidades propedéuticas da poco valor al despertar de la sensibilidad a los aspectos subjetivos y relacionales. Este trabajo pretende resaltar la importancia de la sensibilidad en la educación médica a través de la inclusión de la competencia narrativa en los planes de estudios médicos. Argumentamos que la atención integral y ampliada se construye a través de la integración de la sensibilidad a los elementos biológicos, subjetivos y relacionales del proceso salud-enfermedad-búsqueda de atención, en una trama que da sentido a la vivencia de la enfermedad. Cuanto mejor sepan los clínicos escuchar, absorber e interpretar los informes que les entregan, mejor podrán establecer una conexión que promueva el cambio, cumpliendo con la atención.

\footnotetext{
${ }^{1}$ Psiquiatra e Psicanalista, professora de Psicologia Médica -UFRJ.

${ }^{2}$ Psicóloga, Mestranda em Bioética e Medicina Narrativa pela Universidade Católica Portuguesa.

${ }^{3}$ Professora e pesquisadora - Saúde pública e comunicação em saúde - Universidade Federal Fronteira do Sul.

${ }^{4}$ Psiquiatra, Doutora em Saúde Coletiva - Instituto de Medicina Social, Humanidades e Ciências da Saúde, CHS / IMS-

UERJ, Pesquisador pós-doutorado (IMS-UERJ).
} 
Palabras clave: encuentro clínico, educación médica, empatía, humanidades, medicina narrativa.

\title{
Introdução
}

\begin{abstract}
"Será que o contar de Sabela chegou até mim da maneira que ela falou? Tenho pensado se para um melhor entendimento do que foi... não carece da escuta de outras falas. Quem sabe se, ajuntando pedaços das falas de uns, remendando com o contar de outros, não podia chegar a uma noção mais próxima do realmente acontecido. digo mais próxima porque diante de certos acontecimentos, a palavra é muda. Nem palavra nem gesto dão conta do que verdadeiramente aconteceu." Conceição Evaristo5
\end{abstract}

Há um imaginário de que a produção de conhecimento e prática médica são processos homogêneos, de caráter científico, executados com neutralidade e imparcialidade ${ }^{6,7,8}$. As metáforas ainda predominantes na educação médica, advindas dos pressupostos cartesianos, são mecanicistas (o corpo é uma máquina), lineares (encontrar a causa, criar um efeito) e hierárquicas (o médico visto como um especialista). O cuidado é ainda percebido a partir de uma história de restituição, ou seja, o paciente fica doente e é curado por um médico especialista, ou ainda, sua saúde é restaurada a um estado anterior ao adoecimento. Tais imagens são privilegiadas no cotidiano do ensino e assistência médicos e, por sua vez, influenciam o que é incorporado ao currículo e o que é deixado de lado 9 .

Então podemos perguntar, tendo sido cumpridas as exigências formuladas pela adoção do modelo científico racionalista, como compreender a coexistência de estrondosos aplausos e contundentes queixas dirigidas à Medicina? É voz corrente que a Medicina vai bem, mas os atores da cena assistencial ocupam a posição, alternadamente, de vítimas ou algozes, anjos ou demônios.

Podemos afirmar que nos atendimentos, sem a devida contextualização epidemiológica a proposta racionalista cartesiana foi e continua sendo eficiente e eficaz na resolução da dimensão biológica do adoecer, mas não conseguiu integrar os aspectos subjetivos dos envolvidos nos cenários de prática. Os aspectos emocionais de quem sofre e de quem trata impõem sua presença de forma explícita ou camuflada, com efeitos imediatos, a médio e longo prazo, perceptíveis nos contatos iniciais para elaboração do diagnóstico ou, posteriormente, no encaminhamento das medidas terapêuticas.

\footnotetext{
${ }^{5}$ Conceição Evaristo. Histórias de leves enganos e parecenças (Rio de Janeiro: Malê Editora, 2017$), 84$.

${ }^{6}$ Johanna Shapiro et al., "Medical humanities and their discontents: definitions, critiques, and implications". Academic medicine: journal of the Association of American Medical Colleges, 84, 2 (2009). Doi: https://doi.org/10.1097/ACM.0b013e3181938bca

${ }^{7}$ Rebecca Tsevat et al., "Bringing home the health humanities: narrative humility, structural competency, and engaged pedagogy". Academic medicine: journal of the Association of American Medical Colleges, 90, 11 (2015). Doi: https://doi.org/10.1097/ACM.0000000000000743

${ }^{8}$ Rita Charon et al., The Principles and Practice of Narrative Medicine. (New York: Oxford University Press; 2017).

${ }^{9}$ Johanna Shapiro et al. Ibid.
} 
Quando o público é alcançado por notícias, também aqui sem a devida contextualização epidemiológica, sobre os grandes avanços cirúrgicos, ou sobre potentes fármacos ou sobre o amplo território desbravado pelas práticas de reabilitação e muitas outras realizações, as homenagens se sucedem, exaltando a especificidade do feito, a quantidade de beneficiados e os reflexos sobre a qualidade de vida de muitos. A cena se modifica quando a escuta ou o olhar se voltam para as condições do exercício profissional e para a intimidade da relação profissional de saúde - paciente. Cada vez mais a insatisfação quanto ao relacionamento médico com o paciente e com a comunidade vem sendo registrada na sociedade através de muitos canais de expressão, incluindo a via judicial.

O apelo recorrente às metáforas e narrativas belicistas (luta, batalha, vencer, armas, valentia, resistência, arsenal, estratégia), com pouco espaço para reconhecer ou explorar outras, marginaliza as humanidades e compromete a qualidade da prestação de cuidados ${ }^{10}$. Assim, a necessidade de crítica e construção de práticas inclusivas e éticas é central para o cuidado de qualidade. A medicina envolve quase todos os aspectos da condição humana e é fundamental que estudantes de medicina disponham de ferramentas intelectuais e emocionais adicionais que auxiliem na incorporação da complexidade, ambiguidade e possibilidades presentes no cotidiano do cuidado ${ }^{11}$.

Pode-se dizer que as humanidades médicas têm como objetivo melhorar os cuidados de saúde por meio do refinamento e complexificação dos julgamentos dos médicos em situações clínicas, utilizando como base uma compreensão profunda e complexa do processo saúde-doença-busca de cuidado. Nesse sentido, ainda que alunos e professores apresentem resistências às iniciativas de ensino das humanidades médicas, defendemos que têm um caráter aplicado e essencial na formação médica. Não porque compensa o "biologismo dos cientistas", mas sim porque levam a uma melhor compreensão da condição humana ${ }^{12}$.

As técnicas da medicina narrativa podem auxiliar na diversificação das perspectivas dos alunos de medicina e médicos, permitindo maior tolerância da ambiguidade e questionamento de relações rígidas na educação e prática clínica. Mas, é importante que sejam aplicadas de forma adequada e consciente ${ }^{13,14}$ e para que não sejam desvalorizadas como outras iniciativas das humanidades médicas já foram. Assim, este trabalho tem como objetivos problematizar a noção de sensibilidade na

\footnotetext{
${ }^{10}$ Ibid.

11 Ibid.

12 Ibid.

${ }^{13}$ Rebecca Tsevat et al., "Bringing home the health humanities: narrative humility, structural competency, and engaged pedagogy". Academic medicine: journal of the Association of American Medical Colleges, 90, 11 (2015). Doi: https://doi.org/10.1097/ACM.0000000000000743

${ }^{14}$ Rita Charon et al., The Principles and Practice of Narrative Medicine. (New York: Oxford University Press; 2017).
} 
formação e prática médica e articular essa problematização com a necessidade de inclusão da competência narrativa nos currículos médicos.

Para tanto, a argumentação será desenvolvida em dois tempos. Num primeiro momento, o processo de formação médica calcado no ensino de propedêutica, semiologia e clínica médica, baseados na perspectiva biologicista dos fenômenos saúde-doença, será questionado. Em seguida, desenvolveremos a noção de Competência Narrativa ${ }^{15}$ como estratégia para ampliação da sensibilidade entre alunos e professores, para captar a experiência do adoecer e cuidar dos pacientes e pessoas significativas, relacionar-se interpares, promovendo a coesão das equipes, promover o autocuidado e fortalecer a RMP. Argumentamos que o cuidado integral, ampliado, deve ser construído pela sensibilidade aos elementos biológicos, subjetivos e relacionais do fenômeno saúde-doença-busca de cuidado. Narrar é contar histórias e é através dos enredos vividos que nos percebemos, nos relacionamos e as nossas vidas ganham sentido.

\title{
Formação médica e compreensão biologicista - um outro olhar para o ensino de semiologia e clínica médica
}

\begin{abstract}
"Versões do erro de Descartes obscurecem as raízes da mente humana num organismo biologicamente complexo, mas frágil, finito e único; obscurecem a tragédia implícita no conhecimento dessa fragilidade, finitude e singularidade. E quando os seres humanos não conseguem ver a tragédia inerente à existência consciente, sentem-se menos impelidos a fazer algo para a minimizar"... António Damásio16
\end{abstract}

Os estudos desenvolvidos sobre o ensino médico remetem a estruturação dos procedimentos semiológicos clínicos à percepção do corpo como uma máquina, submetida às leis físicas, como proposto por Descartes (1596-1650). Destaca-se, também, a defesa enfática dos parâmetros cartesianos racionais de obtenção de informações. Na história da filosofia podem ser rastreados outros pensadores que se aproximaram desta temática, mas Descartes elaborou e sistematizou essas proposições ao longo de suas obras filosóficas ${ }^{17}$.

O eixo central do trabalho de Descartes é representado por duas afirmações: no humano existem duas substâncias, matéria e espírito, cabendo à ciência o estudo do corpo/ matéria e à Igreja, o

\footnotetext{
${ }^{15}$ University of Columbia. Rita Charon [Internet]. Disponível em: https://sps.columbia.edu/faculty/rita-charon.

${ }^{16}$ António Damásio. O erro de Descartes - emoção, razão e cérebro humano (Mem-martins: Publicações Europa-América; 1995), 256.

${ }^{17}$ Madel Luz. Natural, racional, social: razão médica e racionalidade científica moderna. (Rio de Janeiro: Campus, 1988), 32.
} 
espírito/ alma; a natureza e seus dinamismos são um objeto criado pela vontade divina entregue à exploração da razão humana ${ }^{18}$.

A proposta filosófica cartesiana é racionalista, definida como tal pelo reconhecimento da razão como única autoridade para se alcançar a verdade e pela eleição do método dedutivo como sua ferramenta básica de trabalho. O exercício racional abre o caminho para se alcançar o verdadeiro conhecimento. Utilizar os sentidos e sentimentos como instrumentos para a obtenção de informações seria assumir o risco de se perder na leitura do mapa capaz de orientar o trajeto em direção à "ciência". O alinhamento aos procedimentos racionais funcionaria como expressão e garantia da assumpção do compromisso com a verdade, uma vez que a metodologia proposta possibilita o controle do que e do como agir, além do como transmitir seus resultados ${ }^{19}$.

Evidencia-se a instalação da oposição ao empirismo então vigente. As premissas de produção do conhecimento a partir da acurada observação do objeto são veementemente rechaçadas. Enquanto os empiristas afirmam que "nada há no intelecto que não tenha estado antes nos sentidos"20, Descartes usa como argumento contrário o amplo espectro de significações atribuíveis a um mesmo dado quando percebido por diferentes observadores. No raciocínio proposto sublinha-se o fato de não ser exequível a reprodução da experiência. Não há como reencontrar a mesma captação do sensível, caracterizado por nuances, em diferentes observadores. E menos ainda, a mesma atribuição de significados.

Sustenta a necessidade de instituir um método e uma linguagem para a produção e comunicação dos saberes com significados inequívocos, como acontece com os conceitos matemáticos. Propõe substituir a qualidade sentida pela quantidade medida ${ }^{21}$ para se alcançar o conhecimento da substância extensa, isto é, o corpo. Fica claro que Descartes não nega a existência da substância anímica, mas endereça seu estudo aos religiosos.

Não demorou para que tal debate chegasse à Medicina. Até o século XVIII, o saber sobre os cuidados dos doentes tinha sido reunido pela experiência acumulada e transmitida às novas gerações por médicos como Hipócrates e Galeno, que haviam se notabilizado por trabalhar com uma perspectiva empírica. Agora, acontece o que Foucault (1998a) denomina de corte epistemológico fundamental na história da medicina ocidental, referindo-se aos movimentos liderados por Pinel (1754 -1826) e por Bichat (1771 - 1826). Enquanto Pinel, a partir do exame de cada doente, busca a analogia dos

\footnotetext{
${ }^{18}$ Madel Luz. Natural, racional, social: razão médica e racionalidade científica moderna. (Rio de Janeiro: Campus, 1988), 49.

${ }^{19}$ Idem.

${ }^{20}$ Hilton Japiassu e Danilo Marcondes. Dicionário Básico de Filosofia. (Rio de Janeiro: Jorge Zahar Editor, 1991$), 79$.

${ }^{21}$ Manuel Morente. Fundamentos de Filosofia. (São Paulo: Mestre Jou, 1970), 174.
} 
sintomas observados em muitos e estabelece os quadros sindrômicos, Bichat trabalha com cortes dos tecidos, chegando à lesão anatômica que determinaria os sintomas descritos clinicamente.

O olhar anátomo-patológico e a anatomoclínica substituem os procedimentos clínicos preconizados por Pinel. Ocorre a transformação do sinal em signo: a queixa trazida pelo paciente sai de cena, as luzes focalizam o fragmento tecidual examinado ao microscópio. Os sintomas clínicos deveriam ser subordinados ao conhecimento da lesão anatômica que os determinou e o funcionamento dos órgãos deverá ser compreendido em termos mecânicos pelas leis físicas. É aplaudida a introdução de aparelhos capazes de aferir os parâmetros físicos. Onde existia o doente com sua singularidade, surge a doença com aspectos observáveis permitindo generalizações ${ }^{22}$.

Mais tarde, Claude Bernard (1813-1878), face à concretude da doença agora "perceptível", introduz a pesquisa sistemática na Medicina com utilização de protocolos de experimentação e métodos de quantificação dos fenômenos fisiológicos. Louis Pasteur (1822-1895) e Robert Koch (18431910) desenvolvem a teoria microbiana das doenças, conduzindo ao conceito de especificidade etiológica.

No cotidiano da assistência, a dimensão subjetiva do sofrimento informada ou percebida no paciente passa a ser considerada um obstáculo a ser transposto, sob pena de comprometer o raciocínio clínico. Pela mesma razão, o médico também deve ignorar os sentimentos nele despertados por seu contato com aquele paciente ou situação. $\mathrm{O}$ âmbito da interdição às expressões predominantemente subjetivas alcança todos os atores envolvidos na cena assistencial. Afirma-se que erros diagnósticos e intervenções terapêuticas inócuas são frutos da contaminação da cena assistencial pela interferência das subjetividades ${ }^{23}$.

A atenção e sensibilidade do profissional devem se dirigir aos dados corporais. Entretanto, as informações obtidas na experiência sensorial vivenciada ao lado do paciente nos procedimentos semiológicos (ver, ouvir, tocar, cheirar) deverão ser submetidas à confirmação pelo exame dos tecidos e células. Manobras de percussão e demais informações obtidas pelo exame físico do paciente permanecem como sinais orientadores da direção a ser seguida na investigação. A sensibilidade do profissional em captar detalhes do funcionamento orgânico com as manobras semiológicas é considerada limitada, inócua ou prejudicial se usada exclusivamente. Os próprios pacientes passam a valorizar a tecnificação do ato médico ${ }^{24}$. Registre-se aqui que empregamos o termo sensibilidade como consta

\footnotetext{
${ }^{22}$ Michel Foucault. O nascimento da clínica. (Rio de Janeiro: Forense Universitária,1998).

${ }^{23}$ Kenneth Camargo Jr. "Paradigmas, ciência e saber médico. Rio de Janeiro." Cadernos do Instituto de Medicina Social. (1992).

${ }^{24}$ Maria Cristina Camargo. "O impacto da ciência e da tecnologia sobre a prática e o ensino médico". (Campinas, Universidade Estadual de Campinas, 1989). Disponível em: 〈http://www.repositorio.unicamp.br/handle/REPOSIP/251967〉.
} 
no dicionário Koogan/Houaiss: substantivo feminino, capacidade de sentir; propriedade de reação dos organismos aos estímulos externos ou internos. E ainda, pelo mesmo dicionário, "sensação: substantivo feminino, impressão recebida pelo sistema nervoso central quando um dos órgãos dos sentidos recebe um estímulo exterior (muitas vezes associado a prazer ou dor)". Acrescentamos que, esquematicamente, esses registros, intermediados pelos sistemas imunológico, hormonal e muscular, podem se fazer presentes em diversas regiões do $\operatorname{corpo}^{25}$.

A sensibilidade é desqualificada e remetida para um papel secundário, enquanto instrumento de captação de informações no âmbito físico, como já o fora em referência aos aspectos subjetivos dos pacientes e médicos. O alinhamento aos dados objetivos é declarado como princípio básico para o exercício da medicina científica e tanto profissionais como pacientes e familiares são convocados a este mister. Mas, por ironia, foi um sentimento, a paixão pela razão que pavimentou a avenida por onde passaram a transitar médicos e pacientes.

Começa a consolidar-se a percepção de que as instituições dedicadas ao ensino médico precisavam se adaptar à nova "medicina científica", afastando tudo que se relacionasse aos pressupostos empíricos. A Associação Médica Americana abraça essa proposta e em 1910, ao final de uma pesquisa realizada nas escolas médicas dos Estados Unidos, é publicado o conhecido Relatório Flexner ${ }^{26}$, defendendo e preconizando uma educação médica pautada no saber biológico e transmitida em ambiente universitário e hospitalar ${ }^{27,28}$. À época, essas eram as exigências a serem cumpridas para se oferecer uma formação médica de boa qualidade. Destacamos os pontos fundamentais das proposições:

- transmissão do saber biológico comprovadamente construído sobre os adoecimentos;

- valorização explícita do alinhamento de todos os docentes ao método científico;

- eleição do ambiente hospitalar como local de excelência para a capacitação clínica;

- vinculação das escolas médicas às universidades, para favorecer a permanente exposição dos estudantes ao pensar e agir dos pesquisadores nas ciências biológicas.

Houve uma adesão generalizada às recomendações de Flexner nas várias partes do mundo onde existiam atividades ligadas à formação dos profissionais da área da saúde. As escolas médicas

\footnotetext{
${ }^{25}$ Andre Koogan e Antonio Houaiss. Enciclopédia e dicionário digital. (São Paulo: Delta, 1998).

${ }^{26}$ Roberto Xavier Piccini, et al. Diretrizes curriculares e o Projeto CINAEM: CINAEM, 2000.

${ }^{27}$ Barbara Barzansky. "Abraham Flexner and the era of medical education reform". Academic medicine: journal of the $\begin{array}{llllll}\text { Association of American Medical Colleges, } & 85, & 9 & \text { (2010): } & \text { S19-S25. } & \text { Doi: }\end{array}$ https://doi.org/10.1097/ACM.0b013e3181f12bd1

${ }^{28}$ Renato Antunes dos Santos e Maria Nunes. "Medical education in Brazil". Medical teacher, 41, 10 (2019): $1106-1111$. Doi: https://doi.org/10.1080/0142159X.2019.1636955
} 
brasileiras que anteriormente seguiam as recomendações de Coimbra, adotaram o modelo flexneriano a partir dos anos cinquenta do século XX, instituindo o curso de medicina estruturado em dois ciclos: o básico, com duração de dois anos e o clínico profissionalizante, realizado em quatro anos.

Vejamos, sucintamente, o alinhamento às proposições do relatório Flexner. No primeiro ciclo, o básico, a proposta é promover a compreensão do processo saúde - doença à luz dos conhecimentos biológicos dispostos em disciplinas tais como Anatomia, Fisiologia, Microbiologia, Genética, Embriologia, Bioquímica, Biofísica, Farmacologia e outras mais. A carga teórica é significativamente alta e as aulas são ministradas por professores/pesquisadores que procuram explicitar a ligação direta ou indireta do conteúdo transmitido com o trabalho assistencial médico. As aulas práticas acontecem nos laboratórios com exposição aos procedimentos e experiências básicas ao cotidiano do trabalho experimental. A frequência aos laboratórios envolvidos, o convívio com pesquisadores e a participação em projetos, são estimulados.

No ciclo profissional, as atividades passaram a ter como cenário os hospitais. A proposta é capacitar o estudante, teórica e praticamente, a realizar intervenções no processo saúde-doença. A metodologia de ensino mantém o modelo aulas teóricas/aulas práticas. No decorrer dos quatro anos, as atividades programadas apresentam características específicas segundo a temática que estiver sendo trabalhada naquele momento. Os primeiros tempos são empregados para capacitar o estudante na Semiologia e Propedêutica Médicas. Segue-se a apresentação das Disciplinas Clínicas e Cirúrgicas, obedecendo à realidade das especialidades médicas praticadas ${ }^{29}$.

Atendendo aos objetivos deste trabalho, nos deteremos na análise dos primeiros anos deste ciclo. As atividades teóricas acontecem como exposições sobre os sinais apreendidos nas práticas semiológicas. Já nas atividades práticas, os estudantes, acompanhados por um supervisor médico e em pequenos grupos, ingressam nas enfermarias, para interagir com pacientes. As enfermarias, geralmente, são unidades de hospitais públicos. Neste cenário iniciam seu treinamento nas manobras semiológicas, além de se apresentarem aos pacientes para colher a história do seu adoecer. Mais adiante, ocorrem as discussões clínicas com o objetivo de estabelecer, a partir das informações colhidas e dos exames realizados, o raciocínio sindrômico. Progressivamente, completa-se o raciocínio clínico propriamente dito - correlacionando etiologia, confirmação de hipótese por exames laboratoriais e linhas gerais da intervenção proposta.

No dia a dia das práticas educativas, professores e estudantes se defrontam com a face viva da Medicina e nos corredores são ouvidos comentários do tipo: "agora sim sinto que entrei em uma

\footnotetext{
${ }^{29}$ Eduardo Marcondes e Ernesto Gonçalves. Educação Médica (São Paulo: Sarvier, 1998).
} 
faculdade de medicina." ..."não sei para que tantas aulas teóricas no básico ..." pensei em desistir como outros colegas fizeram..." "agora sim, serei útil..." Mas ao serem apresentados ao extenso programa de aulas teóricas e avaliações correspondentes, o discurso se modifica... "será que darei conta de aprender tudo de Clínica?" "O que realmente importa para ser um bom médico?"

É perceptível que a relação com o professor se transformará na bússola empregada pelo aluno no vasto continente das informações a ele transmitidas durante o ciclo básico, acrescidas pelas recentemente abordadas no profissional. Mas isso não acontece como um deslizar em mar calmo ao sabor do vento. A direção assumida é resultante da filtragem realizada pelo estudante entre o que foi transmitido em sala de aula, o expresso em palavras pelo supervisor clínico e o que for sendo visto, ouvido e sentido como o real exercício profissional praticado ${ }^{30}$. Acrescente-se que a trama dos fios foi tecida mesclando as experiências de vida do estudante com seu singular modo de ser, pensar e sentir, ainda que suas experiências pessoais não sejam reconhecidas como suporte para o aprendizado ${ }^{31}$.

Consideramos o saber psicanalítico como instrumento de análise pertinente para nos informar sobre o que se passa neste momento da formação do estudante. Em situações de vulnerabilidade e/ou dependência, caracterizadas pelo intenso afluxo de estímulos de várias fontes, como acontece nos primeiros anos de vida dos seres humanos, a restauração do equilíbrio psíquico ameaçado se dá através de processos identificatórios da criança com os personagens fornecedores do cuidado e segurança. Respeitando as especificidades e evitando o reducionismo, numerosos tipos de relações sociais podem ser examinadas com esta lente ${ }^{32}$.

O estudante ao se deparar final e efetivamente com a cena assistencial, percebe como se presentifica o regime de interdições cartesianas quanto à sensibilidade como instrumento de captação da dimensão física e mental dos pacientes:

- papel secundário ocupado pelo exame físico frente aos demais exames pedidos.

- referência à pessoa do paciente propriamente dita somente quando existirem, segundo os critérios do observador, indícios de transtorno mental.

- referência superficial ao relacionamento estabelecido entre paciente e médico, sublinhando sua normalidade. Dificuldades percebidas não devem ser mencionadas.

- igual tratamento merecerão as questões sociais ou quaisquer outras não biológicas.

\footnotetext{
${ }^{30}$ Flávia Terigi. "Notas para uma genealogia do currículo escolar." Educação \& realidade 21, 1 (1996): 159-186.

${ }^{31}$ Janelle Taylor. "Confronting "culture" in medicine's "culture of no culture". Academic medicine: journal of the Association of American Medical Colleges, 78, 6 (2003). Doi: https://doi.org/10.1097/00001888-200306000-00003

${ }^{32}$ Philippe Jeammet, Michel Reynaud, Silla Consoli. Psicologia Médica. Rio de Janeiro: Masson, 1982.
} 
- desvalorização de qualquer registro que escape aos marcos de identificação solicitados pelo censo demográfico.

Esquematicamente poder-se-ia dizer que a adesão ao modelo de assepsia preconizado pela medicina científica é conseguida pelo não feito e pelo não dito, ou seja, as informações pessoais deixam de ser registradas e discutidas, ou, quando são, são de modo superficial ou caricato ${ }^{33}$. Um pacto de silêncio é firmado entre professores e estudantes. As informações ou percepções biológicas são as que realmente fazem parte do raciocínio clínico, merecendo registro nos prontuários e nas aulas.

Médicos e estudantes, quando referem suas experiências como pacientes, salientam, no profissional que os atendeu, competência técnica aliada ao carinho, gentileza, disponibilidade em escutar. Estas qualidades funcionam como a cereja no topo do bolo. Não se reconhece sua participação no raciocínio clínico. O mesmo se aplica quanto às circunstâncias sociais, políticas ou econômicas, envolvidas na situação assistencial. Consequentemente, não se encontram as dimensões psicológica, nem social, nos registros assistenciais. As informações transmitidas pelas disciplinas de cunho humanístico e social não conseguiram legitimar, como se pretendia, a integração das dimensões não biológicas no raciocínio clínico ${ }^{34}$.

Consideramos necessário apontar que as faculdades de medicina foram precocemente alertadas quanto à importância exclusiva atribuída aos aspectos biológicos no currículo médico proposto por Flexner. Acatando essas ponderações, em diferentes momentos históricos, disciplinas de caráter humanístico e social foram sendo incluídas na grade curricular. Mas cumpre dizer, sempre com carga horária restrita e subordinadas ao caráter disciplinar. e invariavelmente precisaram responder aos intermináveis questionamentos sobre seu estatuto de cientificidade. Psicologia Médica, Sociologia Médica, Antropologia Médica, Medicina Preventiva, Saúde Coletiva, Epidemiologia foram alguns dos campos de saber que passaram a constar nos currículos de graduação médica ${ }^{35}$.

Seria inadequado aos objetivos deste artigo detalhar os porquês e as limitações enfrentadas por aquelas disciplinas, mas alguns destaques precisam ser feitos. Sabemos que a especificidade dos objetos de saber e práticas do conhecimento humanístico e social exigem uma vivência primordial

\footnotetext{
${ }^{33}$ Mônica de O. Nunes. "O Silenciamento Da Cultura Nos (con)textos De Cuidado Em Saúde Mental”. Cadernos Brasileiros De Saúde Mental/Brazilian Journal of Mental Health 1, 2 (2011): 31-41.

${ }^{34}$ Johanna Shapiro et al., "Medical humanities and their discontents: definitions, critiques, and implications". Academic medicine: journal of the Association of American Medical Colleges, 84, 2 (2009). Doi: https://doi.org/10.1097/ACM.0b013e3181938bca

${ }^{35}$ Luiz Troncon et al. Conteúdos humanísticos na formação geral do médico (São Paulo: Sarvier, 1998).
} 
para que possa ocorrer, posteriormente, a adequada elaboração. Isso não se alcança com aulas expositivas em ambientes separados dos cenários da prática. Discussão em pequenos grupos sobre o realmente vivido no curso (grupos de reflexão; grupos Balint) a participação dos profissionais de Saúde Mental e Saúde Coletiva nas discussões clínicas, ao longo de todo o curso, foram abrindo novos caminhos constantemente revisitados e elaborados.

É preciso também registrar que, ao longo do tempo, debates acadêmicos têm sido apresentados nos ambientes universitários, explicitando críticas formuladas pela sociedade sobre a qualidade das relações assistenciais praticadas no modelo racionalista. Em muitas dessas discussões, é sublinhado que o raciocínio clínico, predominantemente construído a partir de aspectos anátomo-patológicos, anula a singularidade intrínseca ao humano presente em todos os envolvidos nos processos assistenciais. Outras, igualmente numerosas, apontam o empobrecimento do raciocínio clínico quando os múltiplos determinantes intervenientes no processo saúde-doença deixam de ser considerados, acarretando erros diagnósticos e intervenções desastrosas ${ }^{36}$.

Gradativamente, fomos observando a implementação de várias reformulações dos currículos médicos em todo o mundo e também entre nós, procurando superar as dificuldades apontadas na estrutura curricular. Atualmente, em algumas faculdades, não se privilegia a transmissão do campo de saber das disciplinas, em que um se constitui como base para a seguinte e sim, uma apresentação de conteúdos dados por um conjunto de disciplinas que trabalham integrada e simultaneamente, ou seja, em blocos. Neste segundo modelo, promove-se a multidisciplinaridade no exame dos aspectos biológicos e, com adequação e naturalidade, ocorre o diálogo com os saberes psicológico, social, antropológico e político envolvidos na saúde. Diretrizes curriculares sancionadas com o aval dos órgãos governamentais sustentam a mudança do modelo de educação médica destacando os aspectos biopsicossociais e humanista no sentido amplo do termo, bem como a estratégia da Medicina de Família e Comunidade no planejamento das ações em Saúde Pública ${ }^{37}$.

Retomemos o fio condutor deste artigo para seguirmos adiante. As exigências formuladas pela adoção do modelo científico racionalista foram cumpridas, no entanto, o que se observa é que o método cartesiano para observação e intervenção no fenômeno saúde-doença não apreende importantes aspectos desse processo e não se traduz completamente em bem-estar, qualidade de vida e satisfação com a relação médico-paciente na medicina individual. Como vimos, diversas propostas nas humanidades médicas buscam enfrentar esses desafios. Apresentamos a seguir uma noção que nos parece

\footnotetext{
${ }^{36}$ Kenneth R. Camargo Jr. "Paradigmas, ciência e saber médico. Rio de Janeiro." Cadernos do Instituto de Medicina Social. (1992).

${ }^{37}$ Flávia Terigi. "Notas para uma genealogia do currículo escolar." Educação \& realidade 21, 1 (1996): 159-186.
} 
bastante apropriada para a tarefa de recuperar a sensibilidade na formação e prática médica, e, resgatar seu sentido inicial, o de cuidar.

\section{A Competência Narrativa como estratégia para o despertar e florescer da sensibilidade, ferra- menta de cuidado integral.}

"A alma respira através do corpo, e o sofrimento, quer comece no corpo ou numa imagem mental, acontece na carne" António Damásio38

A Competência Narrativa é central à primeira definição teórica de Medicina Narrativa (MN) apresentada por Rita Charon ${ }^{39,40}$, em que nos ancoramos: "Medicina praticada com a competência narrativa, isto é, a capacidade de reconhecer, absorver, interpretar e ser motivado a agir pelas histórias e dificuldades trazidas pelos outros." 41

Na dimensão da MN como ferramenta da formação médica, que abordamos neste artigo, centramos o nosso olhar no encontro médico. É nesse espaço privilegiado de relação interpessoal e de cruzamento de singularidades que o profissional de saúde é chamado a reconhecer e a dar voz ao sofrimento do paciente e aos significados de que a doença se reveste, ao longo do seu percurso, para ele e para os familiares.

Este movimento de humanização da medicina, que convoca "um modelo de prática clínica informado pela leitura, escrita, narração e escuta de histórias" ${ }^{\$ 2,43}$ integrando também outras linguagens artísticas, tem-se alargado a dimensões como a promoção do autocuidado dos profissionais de saúde (PS), o fortalecimento da relação entre as equipas e a relação com os diversos stake-holders que co-habitam no universo da saúde ${ }^{44}$.

O desenvolvimento das competências narrativas, veiculadas pela leitura atenta e pela escrita reflexiva, mobiliza o poder transformador da expressão de emoções, tanto tempo cerceadas pelo modelo da formação e da prática dos profissionais de saúde. Este dispor-se a tocar e a ser tocado, tendo

\footnotetext{
${ }^{38}$ Antonio Damásio. O erro de Descartes - emoção, razão e cérebro humano (Mem-martins: Publicações EuropaAmérica, 1995): 19.

${ }^{39}$ University of Columbia. Rita Charon [Internet]. Disponível em: https://sps.columbia.edu/faculty/rita-charon

${ }^{40}$ Rita Charon. Doctor-Patient/Reader-Writer: Learning to find the text. Soundings: An Interdisciplinary Journal, 72, 1 (1989): 137-152. Disponível em: http://www.jstor.org/stable/41178470

${ }^{41}$ Rita Charon. The patient-physician relationship. Narrative medicine: a model for empathy, reflection, profession, and trust. JAMA, 286, 15 (2001). Doi: https://doi.org/10.1001/jama.286.15.1897

42 Rita Charon. Honoring the stories of illness [Internet]. USA: TEDxAtlanta; 2011. Disponível em: https://www.youtube.com/watch? $=24 \mathrm{kHX} 2 \mathrm{HtU} 3 \mathrm{o}$

43 Annie Robinson. Narrative Medicine. Madison: University of Wisconsin; 2014. Disponível em: https://www.va.gov/WHOLEHEALTHLIBRARY/docs/Narrative-Medicine.pdf

${ }_{44}$ Maria Giulia Marini. Il portale istud sula medicina narrativa [Internet]. [cited 2020 Oct 18]. Available from: https://www.medicinanarrativa.eu/narrative-medicine
} 
consciência dos seus limites e dos do outro, numa escuta integral e comprometida com uma ética narrativa do cuidado ${ }^{45}$, promove a atenção, a representação e a afiliação, movimentos que constroem a relação com o outro ${ }^{46}$.

A MN, ao indispensável conhecimento especializado que a formação técnico-científica proporciona, procura acrescentar competências que lhe permitam "ler" o paciente-indivíduo, inserido na sua história pessoal única e indissociável do corpo com que se entrega. Pretende resgatar para o encontro médico, nas palavras de João Lobo Antunes, "a atenção às múltiplas cintilações que conferem a cada um de nós a identidade única que é o cerne da dignidade" 47 . Trilhar este caminho para a atenção plena, permitirá acrescentar tempo de qualidade ao tempo cronometrado dos atendimentos, acrescentando camadas de relação e de partilha da subjetividade, que dão espessura ao encontro, construindo a confiança

Reconhecer o outro como ser complexo e multideterminado e deixar-se tocar pelas suas circunstâncias exige também um processo interior de autoconhecimento. "O que sinto é o que da experiência do outro ressoa em mim"48. A MN propõe-se também fazer com os PS este caminho: conhecer-se para melhor cuidar.

Conhecer os nossos vieses (culturais, de classe) e apriorismos, as cicatrizes interiores que nos fazem evitar ou escolher caminhos, as zonas cegas onde não deixamos ninguém entrar, os medos que tolhem à partida o confronto com as nossas próprias vulnerabilidades, desde o logo o confronto com a nossa própria finitude ${ }^{49}$.

Nas escolas médicas, como na prática clínica a MN é, como já referimos, uma ferramenta para o autocuidado:

Trabalhando o confronto com o medo e a ansiedade que inevitavelmente acompanham a tomada de decisão, num contexto em que se tem nas mãos o material mais precioso, a vida de terceiros.

Constituindo-se como um território seguro para antecipar a frustração decorrente do erro médico, limitando danos e retirando dele aprendizagens que construirão oportunidades de melhoria.

\footnotetext{
${ }^{45}$ Susana Magalhães e Joana Araújo. "A Narrativa Ficcional da Deliberação Ética: Olhar a Realidade do Avesso." in 3o Seminário de Bioética, A Decisão em Ética. (Viseu: Comissão de Ética da ESSV, 2011). Disponível em: http://hdl.handle.net/10400.14/9397

${ }^{46}$ Susana Magalhães. "Cuidar do Idoso: medicina narrativa e a arte de demorar-se". Rev Port Bioética, 26, (2020), Disponível em: http://hdl.handle.net/10400.14/31013

${ }^{47}$ João Lobo Antunes (prefácio), In: Atul Gawande. Ser Mortal - Nós, a medicina e o que de verdade importa no final (Alfragide: Grupo Leya; 2015).

${ }^{48}$ Palavras das autoras.

${ }^{49}$ António Damásio. O erro de Descartes - emoção, razão e cérebro humano (Mem-martins: Publicações EuropaAmérica, 1995).
} 
Possibilitando a partilha de experiências interpares, validando e normalizando o que se vivencia.

A medicina, em particular, é uma classe profissional onde continuam a chegar sobretudo aqueles que foram de alguma forma privilegiados no seu percurso de vida. Esta consciência do outro e das suas circunstâncias que a MN pretende trabalhar é também fator de promoção da igualdade no acesso à qualidade dos cuidados de saúde, dando voz aos excluídos, aos que estão nas margens da sociedade, muitas vezes invisíveis num sistema "mainstream", de realidades estanques, com grandes desequilíbrios de poder. Perante uma obra de arte ou perante um texto, analisados numa aula de MN, somos todos seres humanos a sentir e a partilhar a nossa interioridade. Arriscando por a nu a nossa vulnerabilidade e mais disponíveis para a reconhecer naqueles com quem nos cruzamos, em momentos tão íntimos como os encontros em saúde.

A MN pretende treinar a atenção ao detalhe, o colocar-se todo no acto de observar minuciosamente e com todos os sentidos. Escutar integralmente o doente é ouvir o que ele diz, o que esconde, o que gostaria de dizer e não consegue. É escutar e interpretar os silêncios, as metáforas, as fugas, os medos, os interditos, as expectativas, a linguagem do seu corpo, integrar a história da doença na sua história pessoal. É perscrutar a alma no fundo dos olhos e saber comunicar também de corpo inteiro, sendo farol e porto seguro.

A MN pretende assim resgatar a pessoa (o paciente, mas também o médico) para o encontro em saúde e centrar-se na qualidade desse encontro como condição para a co-construção do caminho, integrando a voz do paciente na tomada de decisão.

Existem patologias iguais, mas não existem médicos iguais, pacientes iguais, contextos iguais, pelo que a experiência da doença é sempre diferente, e a sua narrativa co-construída pelos diferentes olhares que a abordam: a história que o doente traz, a que o médico apreende, a que devolve ao doente e a que partilha com os seus pares. Da anamnese, ao diagnóstico. Do prognóstico ao plano de cuidados, passando pelos registros clínicos, tudo constrói o enredo e a narrativa da experiência da doença. A MN pretende ajudar os PS a navegar nas águas da singularidade da relação médico/paciente, a perder o medo de usar as suas emoções e a tirar benefício das emoções em presença no encontro de saúde.

Propõe-se resgatar a capacidade de empatizar, que se perde ao longo do curso de medicina, cujos currículos, assentes em protocolos e saberes livrescos, tem como resultado que os estudantes aprendem a desconfiar dos sentidos, das emoções, da compaixão. 
"the consistent and depressing observation that medical students seem to lose prodigious amounts of empathy as they progress along the medical training route. Something in our medical training system serves to stamp out whatever empathy students bring with them one day"50.

Por empatia, palavra muito gasta e frequentemente mal utilizada, entendemos aqui a atitude que nos dispõe a ver e sentir a partir do ponto de vista do outro, a que a compaixão acrescenta a motivação para agir reduzindo o seu sofrimento, ao ser afetado por ele. A compaixão como a empatia, constutivamente éticas, não são inatas e educam-se, sob pena de na sua ausência caminharmos para uma sociedade moralmente doente.

As ferramentas da MN, nomeadamente a leitura atenta e a escrita reflexiva permitem trabalhar individualmente e em equipe de forma segura para a exposição dos profissionais, dimensões que não podem ser arredadas do encontro clínico, os sentimentos. Só estamos inteiros e comprometidos na relação quando da perceção, chegamos aos sentimentos, passando pelas emoções. Nas palavras de António Damásio "os sentimentos não se limitam a captar meros instantâneos de objetos ou acontecimentos externos; os sentimentos gravam o espetáculo na sua totalidade e a atividade nos bastidores, não só na superfície, mas também no que se encontra abaixo dela"51.

A leitura atenta e orientada de textos literários ou não, de temas médicos ou não, permite aceder a outros olhares e outras formas de ver, desperta para realidades que nos confrontam com as nossas certezas, permite-nos apreender as várias camadas de significados presentes, explorar pistas e referências que alargam os significados e as leituras possíveis. Promove competências de atenção, escuta, tolerância e criatividade pela confrontação com outros pontos de vista. O mesmo se aplica a expressões artísticas como a pintura, o teatro ou a fotografia que são veículo de expressão de emoções.

A partilha de testemunhos interpares, valida sentimentos experienciados e liberta a expressão de emoções, pela sua normalização, eliminando receios de confrontação com valores e códigos institucionais, organizacionais ou de classe. Constrói também a coesão das equipas.

O relato de um médico sobre a sua experiência de doença, como o que a seguir transcrevemos, constitui um bom exemplo do impacto que este tipo de excertos pode ter numa sala de aula e na awareness que despertará:

"Everything seems different as I am in a different role. Lying in bed is a lot more frightening than standing at the end of it in a white coat". Eugene $\mathrm{Wu}^{52}$

\footnotetext{
${ }^{50}$ Danielle Ofri. What Doctors Feel [Internet]. (Boston: Beacon Press; 2013). Disponível em: www.beacon.org

51 António Damásio. Sentir e saber, a caminho da consciência. (Lisboa: Bertrand, 2020). Disponível em: www.temasedebates.pt, 111.

52 Brian Hurwitz, Trisha Greenhalgh, Vieda Skultans. Narrative Research in Health and Illness (Wiley Online Library, 2008), 44.
} 
A escrita reflexiva e particularmente a escrita criativa, permitem ensaiar pontos de vista, vozes, tempos e cenários, reescrever uma história, identificar protagonistas, antagonistas, contextos, outros enredos e outras saídas possíveis (desejadas ou temidas). Sem correr com isso outros riscos, senão o do confronto consigo mesmo, que será sempre fator de crescimento.

Aprender a colocar o outro e as suas circunstâncias num referencial espaço-tempo que é o dele, perceber as suas expectativas, o valor simbólico, estético, quotidiano e instrumental das suas conquistas e das suas derrotas, é essencial para trabalhar a confiança que promoverá a adesão terapêutica e, no limite, a esperança. Os médicos têm que ser preparados para esta liberdade do sentir e não só para a leitura e elaboração de indicadores e relatórios, indispensáveis, mas que não revelam a singularidade do doente por trás da doença, nem permitem cumprir o cuidado integral.

O despertar da sensibilidade e da atenção aos matizes, que a MN promove, fertiliza o terreno onde se desenvolve a criatividade, central para os processos de procura de soluções e de identificação de alternativas, otimizando os processos de tomada de decisão.

Este chão para o ensaio e a reflexão que a MN proporciona, permite também trabalhar a aproximação ao sofrimento alheio entrando na tempestade em modo de segurança, para que o resgate do outro não se transforme no naufrágio do eu. Preservando-se e entregando-se ao mesmo tempo, sendo conscientes dos seus limites e dos sentimentos por trás das emoções em presença.

Permite também, aos futuros médicos, dispor por sua vez de ferramentas que potenciarão a sua forma de recolher e transmitir informação, aumentando a exigência para com outros interlocutores dentro do sistema de saúde, subindo a fasquia da qualidade da comunicação das relações que venham a estabelecer.

O colocar-se à partida o objetivo de conhecer o paciente, o seu contexto e os significados de que a doença se reveste para ele; $\mathrm{O}$ aprimoramento da forma como comunica e se dispõe a escutar integralmente os seus interlocutores, deixando-se tocar pela sua história, sem julgar; O uso judicioso e compassivo das palavras, que são também instrumento de cuidado e contenção do sofrimento do outro. A consciência dos seus próprios limites e vieses bem como do desequilíbrio de poder na relação; A integração da voz do paciente na construção do diagnóstico e do prognóstico, tem enfim como resultado a melhoria da qualidade de prestação dos cuidados de saúde, avaliado pelo melhor e menos tangível dos indicadores: a humanização dos processos.

A MN trabalha a sensibilidade para resgatar e trazer à tona um saber antigo, que tão bem ressoa nas palavras de Rubem Alves ${ }^{53}$ :

\footnotetext{
${ }^{53}$ Rubem Alves. O Médico (Lisboa: Padrões Culturais Editora; 2010), 11.
} 
"noutros tempos os médicos e as enfermeiras sabiam disso. Cuidavam dos remédios e das intervenções físicas - bons para o corpo - mas tratavam de acender a chama misteriosa da alegria. Mas essa chama não se acende com poções químicas. Ela acende-se magicamente, precisa da voz, da escuta, do olhar, do toque do sorriso."

Precisa, diríamos nós, do contributo consciente da nossa irrepetível e frágil humanidade. É isso que a medicina narrativa se propõe acrescentar à exigente formação técnica dos que audaciosamente se propõem ao ofício de ser médico.

\section{Considerações Finais}

Neste trabalho, argumentamos que a sensibilidade para as dimensões subjetivas e relacionais que marcam o encontro clínico é fundamental para o exercício ampliado e ético das práticas de cuidado. A partir das reflexões acerca do ensino médico convencional, baseado num olhar biologicista, foi possível resgatar os papéis da observação e experimentação no método cartesiano e analisar seus desdobramentos na abordagem centrada nos aspectos técnico-científicos privilegiada na medicina moderna. Como foi possível perceber, não se trata somente de limitações ligadas ao modo de examinar os fenômenos inerentes ao processo saúde-doença-busca de cuidado, mas também ao modo de acompanhar aqueles que buscam por cuidados. A visão fragmentada e "cientificizante", em que se baseia o ensino médico, não favorece e contraria até o desenvolvimento de uma atitude empática ${ }^{54}$.

A medicina narrativa, por sua vez, constitui uma abordagem que emprega habilidades narrativas para aumentar a compreensão dos profissionais de saúde quanto às diferentes dimensões do ser humano. Num mundo com contextos sociais e de saúde cada vez mais desafiadores, a medicina narrativa propõe-se ajudar os estudantes de medicina a reconhecer a sua própria voz, proporcionandolhes espaços de reflexão promotores da criatividade, da inovação, da ponderação ética, do exercício do autocuidado.

Propõe-se treinar a escuta das vozes internas, visando integrar no cuidado as dimensões inerentes ao contexto dos indivíduos, que determinam a sua experiência da doença. Para isso, há que aprimorar a sensibilidade quanto à escuta da pessoa promovendo um encontro clínico dialógico entre profissional-paciente. Há que aprender a reconhecer e a gerir o peso específico das palavras, o espaço fecundo dos silêncios e todas as formas de comunicar, da linguagem corporal à expressão artística. Reconhecê-los como ferramentas terapêuticas, redes de cuidado e fundamentos da construção dos encontros clínicos. Defendemos, então, a inclusão da medicina narrativa no âmbito educacional da

\footnotetext{
${ }^{54}$ Maria Giulia Marini. Narrative Medicine Bridging the Gap between Evidence Based Care and Medical Humanities (Milano: Springer International Publishing; 2016).
} 
saúde, como forma de ultrapassar as limitações inerentes ao modelo de formação biologicista e reducionista e, nas palavras de Maria Giulia Marini ${ }^{55}$, "fazer a ponte entre a medicina baseada na evidência e as humanidades médicas", contribuindo para a melhoria da prestação de cuidados de saúde.

\section{Referências Bibliográficas}

Alves Rubens. O Médico. Lisboa: Padrões Culturais Editora; 2010.

Antunes Dos Santos, Renato, Maria Nunes. "Medical education in Brazil". Medical teacher, 41, 10(2019):1106-1111. Doi: https://doi.org/10.1080/0142159X.2019.1636955.

Azeredo, Nára Selaimen, Cristianne Famer Rocha, Paulo Roberto Carvalho. "O enfrentamento da morte e do morrer na formação de acadêmicos de Medicina". Rev Bras Educ Med. 35, 1 (2011):37-43. Doi: https://doi.org/10.1590/S0100-55022011000100006.

Barzansky Barbara. "Abraham Flexner and the era of medical education reform". Academic medicine: journal of the Association of American Medical Colleges, 85, 9 (2010): S19-S25. Doi: https://doi.org/10.1097/ACM.0b013e3181f12bd1

Berger, John. A fortunate man. Traduzido por Pilar Vázquez. Barcelona: Grupo Editorial S.A.U, 2014.

Brasil. Ministério da Educação. Conselho Nacional de Educação. Câmara de Educação Superior. Resolução $\mathrm{N}^{\circ} .3$ de 20 de junho de 2014. Institui diretrizes curriculares nacionais do curso de graduação em Medicina e dá outras providências. Diário Oficial da União, Brasília, 23 jun. 2014; Seção 1, p. 8-11.

Camargo Jr, Kenneth. "Paradigmas, ciência e saber médico. Rio de Janeiro." Cadernos do Instituto de Medicina Social. (1992).

Camargo, Maria Cristina. O impacto da ciência e da tecnologia sobre a prática e o ensino médico. 1989. Dissertação (mestrado) - Universidade Estadual de Campinas, Faculdade de Educação,

Campinas, SP. Disponível em: <http://www.repositorio.unicamp.br/handle/REPOSIP/251967>.

Charon, Rita. "Doctor-patient/reader-writer: learning to find the text." Soundings: An Interdisciplinary Journal, 72(1), 137-152, (1989), http://www.jstor.org/stable/41178470

Charon Rita. The patient-physician relationship. Narrative medicine: a model for empathy, reflection, profession, and trust. JAMA, 286(15), 1897-1902, (2001), https://doi.org/10.1001/jama.286.15.1897

Charon Rita., DasGupta S., Hermann N., Irvine C., Marcus E.R., Colón E.R., Spencer D. The Principles and Practice of Narrative Medicine. New York: Oxford University Press; 2017.

Charon Rita. Honoring the stories of illness [Internet]. USA: TEDxAtlanta; 2011. Disponível em: https://www.youtube.com/watch?v=24kHX2HtU3o

Damásio, Antônio. Sentir e saber, a caminho da consciência. Lisboa: Bertrand, 2020. Disponivel em : www.temasedebates.pt

\footnotetext{
55 Ibid.
} 
Damásio, Antônio. O erro de Descartes - emoção, razão e cérebro humano. Mem-martins: Publicações Europa-América, 1995.

Evaristo, Conceição. Histórias de leves enganos e parecenças. Rio de Janeiro: Malê Editora, 2017.

Foucault, Michel. O nascimento da clínica. Rio de Janeiro: Forense Universitária,1998.

Gawande, Atul. Ser Mortal - Nós, a medicina e o que de verdade importa no final. Alfragide: Grupo Leya, 2015.

Hurwitz, Brian, Trish Greenhalgh, Vieda Skultans. Narrative Research in Health and Illness. Narrative Research in Health and Illness. Nova Jersey: Wiley Online Library, 2008.

Japiassu, Hilton, Marcondes Danilo. Dicionário Básico de Filosofia. Rio de Janeiro: Jorge Zahar Editor, 1991.

Jeammet, Phillipe, Michel Reynaud, Silla Consoli. Psicologia Médica. Rio de Janeiro: Masson, 1982.

Koogan, Abraham, Antonio Houaiss. Enciclopédia e dicionário digital. São Paulo: Delta, 1998.

Luz, Madel. Natural, racional, social: razão médica e racionalidade científica moderna. Rio de Janeiro: Campus, 1988.

Magalhães Susana. "Cuidar do Idoso: medicina narrativa e a arte de demorar-se". Rev Port Bioética, 26, 26 (2020):31-43. Doi: http://hdl.handle.net/10400.14/31013.

Magalhães, Susana, Joana Araújo. "A Narrativa Ficcional da Deliberação Ética: Olhar a Realidade do Avesso". In $3^{\circ}$ Seminário de Bioética, A Decisão em Ética - Resumos. Viseu: Comissão de Ética da ESSV, 2011. Disponível em: http://hdl.handle.net/10400.14/9397

Marcondes, Eduardo, Ernesto Gonçalves. Educação Médica. São Paulo: Sarvier, 1998.

Marini, Maria Giulia. Il portale istud sula medicina narrativa [Internet]. Disponível em: https://www.medicinanarrativa.eu/narrative-medicine

Morente, Manuel Garcia. Fundamentos de Filosofia. São Paulo: Mestre Jou, 1970.

Nunes, Mônica de Oliveira. "O silenciamento da cultura nos (con)textos de cuidado em saúde Mental”. Cadernos Brasileiros De Saúde Mental 1, 2 (2011):31-41. Doi: https://periodicos.ufsc.br/index.php/cbsm/article/view/68472.

Ofri, Danielle. What Doctors Feel [Internet]. Boston: Beacon Press; 2013. Disponível em: www.beacon.org

Piccini, Roberto Xavier, et al. Diretrizes curriculares e o Projeto CINAEM. CINAEM, 2000.

Robinson Annie. Narrative Medicine - Whole Health Library - UW-Madison. Madison: University of Wisconsin, 2014.

Shapiro, Johanna, Jack Coulehan, Delese Wear, Martha Montello. "Medical humanities and their discontents: definitions, critiques, and implications". Academic medicine: journal of the Association of American Medical Colleges, 84, 2 (2009): 192-198. Doi: https://doi.org/10.1097/ACM.0b013e3181938bca

Taylor Janelle. "Confronting "culture" in medicine's "culture of no culture". Academic medicine: journal of the Association of American Medical Colleges, 78, 6 (2003): 555-559. Doi: https://doi.org/10.1097/00001888-200306000-00003 
Terigi, Flávia. "Notas para uma genealogia do currículo escolar." Educação \& realidade 21,1 (1996): 159-186.

Troncon, Luiz E. de A., et al. "Conteúdos humanísticos na formação geral do médico." Em: Marcondes E, Gonçalves EL. Educação Médica. São Paulo: Sarvier, 1998: 99-114.

Tsevat, Rebecca, Anoushka Sinha, Kevin Gutierrez, Sayantani DasGupta. "Bringing home the health humanities: narrative humility, structural competency, and engaged pedagogy". Academic medicine: journal of the Association of American Medical Colleges, 90, 11 (2015), 1462-1465. Doi: https://doi.org/10.1097/ACM.0000000000000743

University of Columbia. Rita Charon [Internet]. Disponível em: https://sps.columbia.edu/faculty/ritacharon 